\title{
10 Schluss
}

Die Akteure einer politisierten Medizin errichteten in den geschlossenen Venerologischen Stationen der DDR Systeme des Terrors und traumatisierten tausende zwangseingewiesene Mädchen und Frauen. Diese Traumatisierungen wirken bis heute und haben teilweise auch Folgen für die Kinder der ehemaligen Zwangseingewiesenen. Bisherige Untersuchungen zu den Auswirkungen politischer Verfolgung in der DDR waren häufig auf die Opfer im Allgemeinen ausgerichtet. Im Mittelpunkt unserer Arbeit stehen Frauen, die Opfer einer politisierten Medizin wurden. Dies begründet die Beschreibung teilweise geschlechtsspezifischer Folgen - unter anderem Traumatisierungen durch gynäkologische Untersuchungen in den geschlossenen Venerologischen Stationen. Die Geschichte der geschlossenen Stationen bildet somit eine weitere Facette der politischen Verfolgung in der DDR, die bereits in der Sowjetischen Besatzungszone (SBZ) beginnt. Die geschlossenen Venerologischen Stationen haben ihren rechtlichen Ursprung in den Befehlen der Sowjetischen Militäradministration in Deutschland (SMAD), die seit Ende des Zweiten Weltkriegs zur Bekämpfung von Geschlechtskrankheiten erlassen wurden. Vor allem der SMAD-Befehl Nr. 273 regelte bis 1961 sämtliche Fragen der Zwangseinweisung von geschlechtskranken und krankheitsverdächtigen Personen. Die Tradition dieser Befehle, Geschlechtskranke oder Krankheitsverdächtige in geschlossenen Einrichtungen zwangseinzuweisen, sie zu disziplinieren, $\mathrm{zu}$ isolieren und sie teilweise zur Arbeit zu verpflichten, wurde in der Gesetzgebung der DDR aufgegriffen. So war in der ,Verordnung zur Verhütung und 
Bekämpfung von Geschlechtskrankheiten“ vom 23. Februar 1961 unter anderem die Zwangseinweisung geschlechtskranker und krankheitsverdächtiger Personen geregelt. Auf dieser rechtlichen Grundlage konnten Menschen, die sich den ärztlichen Auflagen widersetzten, mehrfach als geschlechtskrank registriert wurden oder unter HwG-Verdacht standen, stationär behandelt bzw. in eine geschlossene Station für Geschlechtskranke eingewiesen werden. Zwar war in dieser Verordnung ein dreistufiges Verfahren vorgesehen, an dessen Ende erst die Zwangseinweisung in eine geschlossene Station stand. In der Praxis wurde dieses Gesetz von den Akteuren einer politisierten Medizin allerdings weit ausgelegt oder gar missachtet. So verfügten Kreisdermatovenerologen, Chefärzte oder Fürsorgerinnen häufig die Zwangseinweisungen in geschlossene Venerologische Stationen, ohne das dreistufige Verfahren zu beachten. Ebenso führten Polizisten krankheitsverdächtige oder HwG-verdächtige Personen sofort den geschlossenen Stationen zu und verstießen damit gegen die Verordnung von 1961. Darüber hinaus übergaben Erziehungsheime, Jugendwerkhöfe aber auch Eltern ihre Töchter den geschlossenen Venerologischen Stationen der DDR, wenn sie mit den Kindern nicht zurechtkamen. Solche gesetzeswidrigen Einweisungen lösten bei den Mädchen und Frauen teilweise Schocktraumata aus, wie sie in der Traumaforschung auch für andere politisch Verfolgte der DDR beschrieben werden.

Die offiziellen Zahlen zu den Zwangseinweisungen in die geschlossene Venerologische Station in Halle (Saale) verdeutlichen das Ausmaß der teilweise widerrechtlichen Einweisungen. Wenn 1977 in Halle (Saale) 235 Mädchen und Frauen zwangseingewiesen wurden und diese Zahl einen Trend für die vorangegangenen Jahre darstellt, dann wurden in den 21 Jahren des Bestehens der geschlossenen Venerologischen Station in Halle (Saale) etwa 5.00o Zwangseinweisungen durchgeführt. ${ }^{685}$ Wenn von diesen 5.000 Mädchen und Frauen nur etwa 30\% eine Geschlechtskrankheit hatten, wurden ca. 1.500 geschlechtskranke sowie ca. 3.50o gesunde Personen zwangseingewiesen, diszipliniert, isoliert, (medizinisch) behandelt und in der Folge traumatisiert. Die DDR-weite Dimension wird durch die Zahlen zu den Zwangseinweisungen aus dem Jahr 1968 deutlich: In diesem Jahr wurden in den 15 Bezirken der DDR insgesamt 2.763 Personen wegen des Verdachts auf eine Geschlechtskrankheit zwangseingewiesen. ${ }^{686}$ Auch 1968 konnte lediglich bei 777 Personen (28\%) eine Erkrankung nachgewiesen werden, sodass bei etwa 70\% keine Geschlechtskrankheit vorlag. Würde man die 2.763 Zwangseinweisungen aus dem Jahr 1968 mit den 40 Jahren des Bestehens der DDR multiplizieren, käme man auf eine sechsstellige Zahl an Zwangseinweisungen für die gesamte DDR. Diese Zahl würde dann aber noch immer nicht die Zwangseinweisungen in der SBZ beinhalten. Es wird an dieser Stelle deutlich, wie hoch die Zahl der Betroffe-

685 Münx, Bruns, Rudolf: Venerische Erkrankungen und ihre Prophylaxe (Anm. 10), S. 72.

686 Elste, Krell: Zur Bedeutung der Verhütungs- und Bekämpfungsmaßnahmen (Anm. 17), S. 937. 
nen ist, die bis heute an den Folgen der Zwangseinweisungen leiden bzw. wegen der traumatischen Erlebnisse in therapeutischer Behandlung sind.

Die rechtlichen Bedingungen der „Verordnung zur Verhütung und Bekämpfung von Geschlechtskrankheiten“ wurden nicht nur im Rahmen der Einweisung, sondern auch hinsichtlich der Funktion und der Gestaltung des Alltags in den geschlossenen Venerologischen Stationen von einer politisierten Medizin weit ausgelegt. Ohne Vorgaben des Ministeriums für Gesundheitswesen erließen Chefärzte Hausordnungen für ihre jeweilige geschlossene Station und legten damit ein internes Reglement fest. Besonders deutlich wurde das Handeln einer politisierten Medizin in Halle (Saale). Mit der Unterbringung auf der geschlossenen Venerologischen Station sollten die geschlechtskranken und „asozialen“ Personen durch erzieherische Einwirkung zu einer „sozialistischen Persönlichkeit“ erzogen werden, wie es in der Hausordnung von Halle (Saale) heißt. Auch die Aufnahme war in der Hausordnung geregelt und wurde von den Zeitzeuginnen wie folgt beschrieben: Entkleidung, Abnahme persönlicher Gegenstände, Waschen (ggf. mit Rasur), Ankleiden mit Anstaltskleidung (blauer Kittel), Anamnese, gynäkologische Untersuchung und Zuweisung eines Bettes. Bei der Aufnahme der Mädchen und Frauen wurde das medizinisch-pflegerische Personal oft von den Stubenältesten unterstützt. Die Anamnese, bei welcher auch Angaben zu Sexualpartnern gemacht werden mussten, wurde anfänglich von Famulanten, später von einem Psychologen erhoben. Die gynäkologischen Untersuchungen führten im Wesentlichen die Stationsärzte bzw. die Schwestern durch. Schließlich war selbst der Tagesablauf auf der Station in der Hausordnung geregelt und wurde von den Zeitzeuginnen bestätigt: Die Zwangseingewiesenen wurden vor 6 Uhr geweckt. Es folgten anschließend das Waschen, die tägliche gynäkologische Untersuchung und das Frühstück. Jede arbeitsfähige Patientin war in Halle (Saale) zur sogenannten „Arbeitstherapie“ verpflichtet. Die Zwangseingewiesenen konnten zu Reinigungsarbeiten der Krankenzimmer bzw. Krankenhausräume, aber auch in anderen Objekten der Poliklinik Mitte herangezogen werden.

Vor allem die täglichen gynäkologischen Untersuchungen, die für alle geschlossenen Venerologischen Stationen in der DDR belegt sind, führten zu Traumatisierungen. Sie wurden gegen den Willen der Mädchen und Frauen durchgeführt. In den meisten Fällen (70\% der Zwangseingewiesenen) lag keine medizinische Indikation, kein tatsächlicher körperlicher Befund vor. Zur Entnahme des Abstrichs, so beschreiben es die Zwangseingewiesenen, wurde ihnen ein Glasrohr vaginal eingeführt. Dabei kam es häufig zu Verletzungen, Blutungen und bei Erstabstrichen zu Deflorationen. Wehrten sich die Mädchen und Frauen, dann wurden sie von der Stubenältesten festgehalten. Vor allem bei jungen Mädchen wurden die Abstriche im Sinn einer abschreckenden und disziplinierenden Maßnahme eingesetzt. Waren die Abstriche bei Erstabstrich negativ, wurden bei den Mädchen und Frauen Provokationsmaßnahmen vorgenommen. Mithilfe von Fieber auslösenden Mitteln sollten mögliche Infektionen getriggert und eine Infektion zum Vorschein gebracht wer- 
den. Die unmittelbare Folge waren Übelkeit, hohes Fieber und schubweise auftretende Krämpfe am gesamten Körper. Lagen tatsächlich Geschlechtskrankheiten vor, wurden sie mit Penicillin behandelt. Der Behandlungszeitraum betrug in Halle (Saale) vier bis sechs Wochen. Die medizinischen Eingriffe wurden ohne Aufklärung und ohne Einwilligung der Zwangseingewiesenen durchgeführt. Die Spätfolgen der gynäkologischen Untersuchungen sind unter anderem Angst vor Gynäkologen und Ärzten generell, sexuelle Inappetenz und Beziehungslosigkeit.

Auf der Station in Halle (Saale) herrschte ein hierarchisches Terrorsystem, das in der Hausordnung vorgegeben und vom medizinisch-pflegerischen Personal sowie den Stubenältesten umgesetzt wurde. Der Terror bestand aus einem System von Belobigungen und Disziplinarmaßnahmen. Zu den Belobigungen zählten die Erteilung zusätzlicher Raucherlaubnis, die Löschung einer Hausstrafe oder schriftliches Lob. Disziplinarmaßnahmen waren die zusätzliche "Arbeitstherapie“, „Abstrichsperre“ sowie die Nachtruhe außerhalb des Bettes auf einem Hocker. Diese Maßnahmen, so berichten die Zeitzeuginnen, wurden häufig angewandt und dienten der Erziehung der Zwangseingewiesenen. Der Leiter der Station und das medizinisch-pflegerische Personal waren unpersönlich, entindividualisierend und misshandelten gemeinsam die Zwangseingewiesenen durch gezieltes Zufügen von Schmerzen während der Abstriche. Gleichzeitig disziplinierten Ärzte und pflegerisches Personal die Zwangseingewiesenen durch Belohnungen (Zuteilung von Zigaretten) und Bestrafungen (Schlafentzug). Dieses Terrorsystem wurde zusätzlich durch die Stubenältesten stabilisiert, welche ebenfalls als Zwangseingewiesene auf der geschlossenen Station waren. Sie teilten den Stubendienst ein, organisierten die Hilfstätigkeiten oder mussten die Strafen (24 Stunden Isolation, Hockersitzen oder Schlaf- und Zigarettenentzug) überwachen. Einige Zwangseingewiesene berichten, dass sie auf der Station Widerstand geleistet hätten. Dieser Widerstand wurde aber stets durch das medizinisch-pflegerische Personal sanktioniert. Ein Opponieren durch Angehörige der Mädchen und Frauen oder durch das Personal ist nicht überliefert. Die geschlossene Venerologische Station in Halle (Saale) wurde seit 1976 durch das Ministerium für Staatssicherheit (MfS) überwacht. Aus den Akten des Bundesbeauftragten für die Unterlagen des Staatssicherheitsdienstes der DDR (BStU) geht hervor, dass das MfS Einfluss auf die Entwicklungen in der Poliklinik Mitte bzw. der geschlossenen Venerologischen Station in Halle (Saale) nahm und einen wesentlichen Beitrag an der Auflösung der geschlossenen Venerologischen Station hatte.

Im Vergleich der geschlossenen Venerologischen Station in Halle (Saale) mit weiteren Einrichtungen in der DDR überwiegen die Gemeinsamkeiten. Wie in Halle (Saale) wurden auch in Leipzig-Thonberg die 12- bis 7ojährigen Frauen aus allen sozialen Schichten zwangseingewiesen. Die Einweisungen waren in der Art und der Form in Halle (Saale) und in Leipzig-Thonberg identisch. Anders als in Halle (Saale) führten die gesetzeswidrigen Einweisungen in die geschlossene Venerologische Station in Leipzig-Thonberg zu Ermittlungen der 
Staatsanwaltschaft von Leipzig. Diese Ermittlungen hatten kaum Änderungen zur Folge, wie die Aussagen der Zeitzeuginnen bestätigen. Gemeinsamkeiten zwischen Halle (Saale) und Leipzig-Thonberg bestanden auch in der Prozedur der Aufnahme und in der entindividualisierenden Behandlung der Patientinnen. Dies gilt auch für die medizinischen Eingriffe, die in Leipzig-Thonberg nahezu identisch, ohne Aufklärung und ohne Einwilligung der Zwangseingewiesenen durchgeführt wurden. In Halle (Saale) wie auch in Leipzig war der Tagesablauf durch eine Hausordnung geregelt. Auch die interne Hierarchie (Ärzte, Pflegerinnen, Stubenälteste), die in Halle (Saale) den Terror im Terror ermöglichte, war in Leipzig-Thonberg wiederzufinden. Trotz mehrerer Trägerwechsel wurde die bauliche Gestaltung der geschlossenen Venerologischen Station Leipzig-Thonberg (vergitterte Fenster und verschlossene Türen) nie verändert oder aufgehoben. Wie in Halle (Saale) war auch die geschlossene Venerologische Station Leipzig-Thonberg nach außen hin abgeschottet und isolierte die Zwangseingewiesenen von der Außenwelt. Entsprechend beschreiben die ehemals zwangseingewiesenen Frauen die gleichen Spätfolgen und sind von den gleichen traumatischen Ereignissen bis heute geprägt. Es gab aber auch einige Unterschiede zwischen Halle (Saale) und Leipzig-Thonberg. Während die geschlossene Venerologische Station in Halle (Saale) aus einer 1951 eingerichteten „Beobachtungs- und Fürsorgestation“ für Geschlechtskranke hervorging, hat die Station in Leipzig-Thonberg eine umfangreiche Vorgeschichte, die bis ins 19. Jahrhundert reicht. Vor allem das 1946 eingerichtete Fürsorgeheim Leipzig-Thonberg, das Gemeinsamkeiten mit dem Fürsorgeheim in Zwickau aufwies, prägte die geschlossene Venerologische Station Leipzig-Thonberg. Übernommen wurden aus dem Fürsorgeheim die Innenausstattung, die Pflicht zur Arbeit sowie die Isolation. Deutlich verschieden waren auch die Kapazitäten. Während in Halle (Saale) ca. 30 Betten zur Verfügung standen, konnten in Leipzig-Thonberg in den 196oer Jahren etwa 6o Betten belegt werden. Erst in den 1970er Jahren wurde die Anzahl der Betten in Leipzig-Thonberg auf ca. 30 reduziert. Ein weiterer Unterschied zwischen Halle (Saale) und Leipzig-Thonberg bestand in der „Arbeitstherapie“. Während in Halle (Saale) von der Gründung bis zur Auflösung der Station eine Pflicht zur Arbeit bestand, wurde Mitte der 1970er Jahre in Leipzig-Thonberg aus der Pflicht die Möglichkeit zur Beschäftigung. Seit dieser Zeit wurden die zwangseingewiesenen Mädchen und Frauen in Leipzig-Thonberg weniger diszipliniert, sondern vor allem verwahrt. Dies wird auch daran deutlich, dass in Leipzig-Thonberg weniger Sanktionen herrschten.

Neben der geschlossenen Venerologischen Station in Halle (Saale) und in Leipzig-Thonberg waren in Berlin und in Berlin-Buch geschlossene Stationen eingerichtet. Auch in Berlin bzw. später in Berlin-Buch wurden Mädchen und Frauen durch die Polizei zugeführt und von den verantwortlichen Venerologen zwangseingewiesen. Sowohl in der Art und Form der Zwangseinweisungen, Aufnahmen, medizinischen Eingriffe, der Gestaltung der Stationen (vergitterte Fenster und Türen) als auch in der internen Hierarchie finden sich keine 
Unterschiede zwischen den geschlossenen Stationen in Berlin, Berlin-Buch, Halle (Saale) und in Leipzig-Thonberg. Unterschiede bestehen vor allem in drei Punkten. Zum einen war auch in Berlin der Tagesablauf klar strukturiert. Dennoch wurden die Zwangseingewiesenen in Berlin und Berlin-Buch nicht beschäftigt, sondern verwahrt und asyliert. Zum anderen wurde die Verwahrung ab 1977 durch einen Ausbau der Sicherungsmaßnahmen intensiviert. Diese Sicherungsmaßnahmen dienten der Isolation und Disziplinierung der zwangseingewiesenen Mädchen und Frauen. Darüber hinaus unterschied sich die geschlossene Venerologische Station Berlin-Buch von allen anderen untersuchten Stationen in der Durchführung von Kosmetiktests. Seit 1964 gehörten Kosmetikartikel zu den Gesundheitspflegemitteln und fielen damit unter das Arzneimittelgesetz der DDR. Entsprechend musste deren Unbedenklichkeit getestet werden. Diese Tests wurden unter anderem an den zwangseingewiesenen Mädchen und Frauen in Berlin-Buch ohne Aufklärung und Einwilligung durchgeführt. Dadurch wurden Hauterkrankungen provoziert und Aggressionen unter den Zwangseingewiesenen verstärkt.

Schließlich ist für die geschlossene Venerologische Station in Dresden-Friedrichstadt belegt, dass auch sie Vorgängereinrichtungen hatte, die bis zum Anfang des 20. Jahrhunderts zurückreichen. Die Patientencharakteristik, die Art und Form der Zwangseinweisung, der Aufnahme, der medizinischen Eingriffe, der Gestaltung der Stationen, der internen Hierarchie als auch die traumatisierenden Ereignisse waren mit den geschlossenen Stationen in Berlin, Berlin-Buch, Halle (Saale) und Leipzig-Thonberg identisch. Letztlich wurde die Auflösung der geschlossenen Venerologischen Station in Dresden-Friedrichstadt durch einen Wechsel des Chefarztes 1976 eingeleitet und umgesetzt, der keine Notwendigkeit in einer geschlossenen Station sah.

Die Venerologischen Stationen in Magdeburg und Zwickau waren nach unserem aktuellen Forschungsstand keine geschlossenen Stationen. Obwohl in diesen Stationen Zwangseinweisungen durchgeführt wurden, waren diese beiden Stationen wesentlich durchlässiger (Besuchszeiten, geringe Isolation). Hier besteht aus unserer Sicht weiterer Forschungsbedarf. Wichtig sind neben vertieften Archivrecherchen vor allem weitere Interviews mit Zeitzeugen. Weiterer Forschungsbedarf besteht auch hinsichtlich der geschlossenen Venerologischen Stationen in Erfurt, Rostock und Schwerin. An diesen drei Standorten existierten in den 196oer Jahren geschlossene Stationen, die aus unterschiedlichen Gründen - unter anderem aus Personalmangel - aufgelöst wurden. Wichtig für die weitere Forschung ist zudem ein Ost-West-Vergleich. Ein solcher Vergleich stellt sich vor allem vor dem Hintergrund der unterschiedlichen Entwicklungen in der SBZ und den anderen Besatzungszonen der Alliierten als notwendig dar. So wurden in der SBZ andere Traditionen im Umgang mit Geschlechtskranken entwickelt, die bis spät in die DDR nachgewirkt haben. Voraussetzung hierfür ist die Untersuchung der Praxis der Zwangseinweisung und Behandlung von geschlechtskranken Personen in der Bundesrepublik Deutschland. 
In den von uns untersuchten geschlossenen Venerologischen Stationen wurden Mädchen und Frauen zwangseingewiesen, diszipliniert und isoliert. Teilweise waren die Zwangseingewiesenen zur Arbeit verpflichtet. Der Aufenthalt in den geschlossenen Stationen hatte sowohl unmittelbare Folgen als auch Spätfolgen in Form von schweren Traumatisierungen. So wurden im Rahmen gynäkologischer Untersuchungen teilweise frühkindliche Erfahrungen mit sexueller Gewalt reaktualisiert. Aber auch jene Frauen, die während der Kindheit keine sexuelle Gewalt erfahren haben, beschreiben die täglichen medizinischen Eingriffe in ihre körperliche Integrität als schockierend und traumatisierend. In der Folge stellten sich bei den zwangseingewiesenen Frauen häufig sexuelle Inappetenz ein. Vielen ehemaligen zwangseingewiesenen Frauen fiel im späteren Leben die Bindung zu einem Partner schwer. Sie versuchten, nach dem Aufenthalt in den geschlossenen Stationen Beziehungen zu Männern aufzubauen, gründeten schnell eine eigene Familie und verließen sie häufig rasch wieder. Stattdessen versuchten sie, durch schnelle Partnerwechsel ein neues Zuhause zu finden. Einige Frauen, die in ihrem späteren Leben eine feste Bindung eingegangen sind und ihre Partnerschaft als glücklich beschreiben, zweifeln bis in die Gegenwart an der Dauerhaftigkeit der Beziehung und ihrer eigenen Beziehungsfähigkeit. Sie beschreiben ein grundsätzliches Unwohlsein im Zusammensein mit einem männlichen Partner. Spätfolgen wie sexuelle Inappetenz und Schwierigkeiten in den sozialen Bindungen bestimmen nach der Entlassung aus den geschlossenen Venerologischen Stationen das weitere Leben der zwangseingewiesenen Frauen.

Im Gegensatz zu den Meinungen von Ärzten, Pflegepersonal oder Fürsorgerinnen, welche die Zwangseingewiesenen als „Rumtreiberinnen“ oder als „Arbeitsbummelantinnen“ beschrieben, hatte die Arbeit im Leben der zwangseingewiesenen Frauen einen hohen Stellenwert. Sie arbeiteten vor und nach der Einweisung in ihren Betrieben und wechselten den Arbeitsplatz auch nach dem Aufenthalt in den geschlossenen Venerologischen Stationen nicht. Viele waren in Drei- und Vier-Schicht-Systemen beschäftigt und arbeiteten nach eigenen Angaben bis zu zwölf Stunden täglich. Diese umfassende Berufstätigkeit hatte Folgen für die Kinder der Zwangseingewiesenen. Die Kinder wurden in Wochenkrippen und Betriebskindergärten, von staatlichen Erzieherinnen betreut, während die alleinerziehenden Mütter im Schichtsystem arbeiteten. Vor diesem Hintergrund konnten Mutter-Kind-Beziehungen nur schwer aufgebaut werden. Waren die Mütter alleinerziehend, wurden die Kinder häufig in Heimen untergebracht, wenn die Mütter in die geschlossenen Stationen zwangseingewiesen wurden. In einigen Fällen wurden die Kinder sogar zur Adoption freigegeben. Meist verschwiegen die Fürsorgeeinrichtungen und Adoptiveltern den Kindern den eigentlichen Aufenthaltsort ihrer Mütter. Auch die zwangseingewiesenen Frauen schwiegen nach ihrer Entlassung und sagten den Kindern nicht, wo sie gewesen waren. Die teilweise beschriebene Vernachlässigung der Kinder aber auch das Schweigen über den Zwangsaufenthalt in den geschlossenen Stationen hatten unterschiedliche Folgen für die 
Kinder: Sie entwickelten ein Misstrauen gegenüber den Biographien der Mütter oder lösten sich im Erwachsenenalter vollständig von ihren Müttern; teilweise bildeten sie Suchterkrankungen aus.

Viele der ehemaligen Zwangseingewiesenen Frauen werden therapeutisch begleitet. Einige Frauen versuchen ihre Traumatisierungen unter anderem durch künstlerisches Tätigsein zu bearbeiten. So haben einige ihre Lebensgeschichte aufgeschrieben und die Erlebnisse in den geschlossenen Venerologischen Stationen dargestellt. Neben der Autobiographie, die Juli Sommermond vor einigen Jahren geschrieben hat, ${ }^{687}$ verfasste Bettina Weben das Buch „Gequält, Erniedrigt und doch am Leben: Warum war die DDR so ungerecht?", in dem sie unter anderem auf die Zeit in der geschlossenen Venerologischen Station in Halle (Saale) eingeht. ${ }^{688}$ Des Weiteren beschäftigt sich ein Theaterprojekt in Berlin mit der künstlerischen Bearbeitung der Ereignisse auf der geschlossenen Venerologischen Station in Berlin-Buch. Gemeinsam setzten sich die ehemaligen zwangseingewiesenen Frauen für die Anerkennung des an ihnen begangenen Unrechts ein. Die Rehabilitation der Opfer ist keine Aufgabe, die vonseiten der Wissenschaft initiiert werden kann, wohl aber begleitet und unterstützt. Fragen der Rehabilitation gehören vor allem in den politischen Raum. Unsere Forschungsarbeit zu den geschlossenen Venerologischen Stationen in der DDR hat gezeigt, dass solche Stationen ein DDR-weites Phänomen waren, sodass die Landesparlamente aller neuen Bundesländer aufgefordert sind, sich für die Rehabilitation einzusetzen, an den Bund heranzutreten und den rechtlichen Rahmen für mögliche Rehabilitationen voranzubringen.

Gewidmet ist das Buch den Frauen.

687 Sommermond I (2013) Tod einer Kinderseele. Waidhaus.

688 Weben B (2015) Gequält, Erniedrigt und doch am Leben: Warum war die DDR so ungerecht? Books on Demand Norderstedt. 